\title{
On CM-fields with the same maximal real subfield
}

\author{
by
}

\author{
Kuniaki Horie (Hiratsuka)
}

We shall mean by a number field a finite extension over the rational field $\mathbb{Q}$ contained in the complex field $\mathbb{C}$, and by a $C M$-field a totally imaginary quadratic extension in $\mathbb{C}$ over a totally real number field. Let $k$ be a totally real number field. Let $\Gamma$ denote the set of all CM-fields that are quadratic extensions over $k$, so that $\Gamma$ is an infinite set. In this paper, giving a characterization of CM-fields with odd relative class number, we shall prove that there exist infinitely many CM-fields in $\Gamma$ with odd relative class number if and only if the class number of $k$ in the narrow sense is odd. We shall also find out, by virtue of formulae of Kida [7], when $\Gamma$ contains infinitely many CM-fields $K$ such that $\mu_{K}^{-}=\lambda_{K}^{-}=0$. Here, for each CM-field $K, \mu_{K}^{-}$and $\lambda_{K}^{-}$denote respectively the Iwasawa $\mu^{-}$- and $\lambda^{-}$-invariants associated with the basic $\mathbb{Z}_{2}$-extension over $K, \mathbb{Z}_{2}$ being of course the additive group of the 2-adic integer ring. An additional remark will be made in the last section.

Part of the notation used in the paper is as follows. For any number field $F$, we let $C_{F}$ denote the ideal class group of $F, A_{F}$ the Sylow 2-subgroup of $C_{F}$, and $h_{F}$ the class number of $F ; h_{F}=\left|C_{F}\right|$. Moreover, $h_{F}^{*}$ will denote the class number of $F$ in the narrow sense, $F^{+}$the maximal real subfield of $F, I_{F}$ the ideal group of $F, E_{F}$ the unit group of $F, E_{F}^{*}$ the subgroup of $E_{F}$ consisting of all totally positive elements of $F$ in $E_{F}$, and $N_{F / H}$, for each subfield $H$ of $F$, the norm map from the multiplicative group $F^{\times}=F \backslash\{0\}$ to the multiplicative group $H^{\times}$. Given arbitrary algebraic numbers $\alpha_{1}, \ldots, \alpha_{n}$ in $\mathbb{C}$, we write $\left(\alpha_{1}, \ldots, \alpha_{n}\right)$ for the fractional ideal of $\mathbb{Q}\left(\alpha_{1}, \ldots, \alpha_{n}\right)$ generated by $\alpha_{1}, \ldots, \alpha_{n}$. It is therefore understood that $\left(\alpha_{1}, \ldots, \alpha_{n}\right)$ lies in $I_{F}$ whenever the number field $F$ contains $\alpha_{1}, \ldots, \alpha_{n}$. Now, let $K$ be any CM-field. We then denote by $A_{K}^{-}$the Sylow 2-subgroup of the kernel of the norm map $C_{K} \rightarrow C_{K^{+}}$and by $h_{K}^{-}$the relative class number of $K ; h_{K}^{-}=h_{K} / h_{K^{+}}$. As is well known, the norm map $C_{K} \rightarrow C_{K^{+}}$is surjective so that $h_{K}^{-}$equals the order of the kernel of this norm map. We let $t_{K}$ denote the number of prime ideals of $K$ ramified for $K / K^{+}$. The rank of each finite abelian group $G$ will be denoted by $r(G)$. 
1. We first give a brief proof of the following fact which might be essentially well known.

Lemma 1. Let $K$ be a $C M$-field such that $2 \nmid h_{K^{+}}$. Then

(i) $t_{K}-1 \leq r\left(A_{K}\right) \leq t_{K}-1+\left[K^{+}: \mathbb{Q}\right]-r\left(E_{K^{+}} / E_{K^{+}}^{*}\right)$,

(ii) $r\left(A_{K}\right)=t_{K}-1+\left[K^{+}: \mathbb{Q}\right]-r\left(E_{K^{+}} / E_{K^{+}}^{*}\right)$ if $t_{K}=0$ or 1 .

Pr o o f. By $2 \nmid h_{K^{+}}$, the ambiguous ideal classes for $K / K^{+}$in $A_{K}$ coincide with the ideal classes in $A_{K}$ of order at most 2. The ambiguous class number formula (cf. Satz 13 in Ia of [4]) therefore implies

$$
r\left(A_{K}\right)=t_{K}-1+\left[K^{+}: \mathbb{Q}\right]-r\left(E_{K^{+}} /\left(N_{K / K^{+}}\left(K^{\times}\right) \cap E_{K^{+}}\right)\right) .
$$

Thus (i) follows from

$$
E_{K^{+}}^{*} \supseteq N_{K / K^{+}}\left(K^{\times}\right) \cap E_{K^{+}} \supseteq E_{K^{+}}^{2}=\left\{\varepsilon^{2} \mid \varepsilon \in E_{K^{+}}\right\} .
$$

Next assume $t_{K}=1$. The product formula for the Hasse norm residue symbol then shows that every element of $E_{K^{+}}^{*}$ is a norm residue for $K / K^{+}$ modulo the conductor of $K / K^{+}$, whence, by the Hasse norm theorem for $K / K^{+}$,

$$
E_{K^{+}}^{*} \subseteq N_{K / K^{+}}\left(K^{\times}\right), \quad \text { namely } \quad E_{K^{+}}^{*}=N_{K / K^{+}}\left(K^{\times}\right) \cap E_{K^{+}} .
$$

Therefore from (1.1) we obtain

$$
r\left(A_{K}\right)=\left[K^{+}: \mathbb{Q}\right]-r\left(E_{K^{+}} / E_{K^{+}}^{*}\right)
$$

as stated in (ii).

In the case $t_{K}=0$, the assertion (ii) is an immediate consequence of (1.1) and the Hasse norm theorem for $K / K^{+}$.

By the Hilbert 2-class field over a number field $F$, we shall mean as usual the maximal unramified abelian 2-extension over $F$ in $\mathbb{C}$.

Theorem 1. Let $K$ be a $C M$-field. Let $M$ denote the Hilbert 2-class field over $K^{+}$. Then $h_{K}^{-}$is odd if and only if the following conditions are satisfied:

(1-i) $M$ is cyclic over $K^{+}$, i.e., $A_{K^{+}}$is cyclic,

(1-ii) $t_{K}=0$ or 1 and, in the case $t_{K}=1$, the prime ideal of $K^{+}$ ramified in $K$ remains prime in $M$,

(1-iii) $r\left(E_{M} / E_{M}^{*}\right)=[M: \mathbb{Q}]+t_{K}-1$.

Proof. We note first of all that the inequality $r\left(A_{K}^{-}\right)+1 \geq r\left(A_{K^{+}}\right)$ holds in general (cf. Proposition 10.12 of [12]).

Now, assume that $h_{K}^{-}$is odd, so that (1-i) certainly holds by the above inequality. Obviously the composite $K M$ is a CM-field whose maximal real subfield is $M$. Since $h_{M}$ is odd by (1-i), we see from Lemma 1 that $1 \geq$ 
$t_{K M}=t_{K}$, which implies (1-ii). Lemma 1 further shows us that

$$
t_{K}-1+[M: \mathbb{Q}]-r\left(E_{M} / E_{M}^{*}\right)=r\left(A_{K M}\right) .
$$

However, the right hand side here equals 0 by $A_{K}^{-}=\{1\}$ or equivalently by $[K M: K]=\left|A_{K}\right|$, because $K M$ is the Hilbert 2-class field over $K$ as well as a cyclic extension over $K$. Thus we also have (1-iii).

Let us next assume (1-i)-(1-iii). As $h_{M}$ is odd by (1-i) and as $t_{K}=$ $t_{K M} \leq 1$ by (1-ii), it follows from Lemma 1 and (1-iii) that

$$
r\left(A_{K M}\right)=t_{K}-1+[M: \mathbb{Q}]-r\left(E_{M} / E_{M}^{*}\right)=0 .
$$

Hence we have $r\left(A_{K}^{-}\right)=0$, i.e., $2 \nmid h_{K}^{-}$. Theorem 1 is therefore proved.

EXAMPLE. Let $p$ be a prime number $\equiv 5(\bmod 8)$. Then $\mathbb{Q}(\sqrt{2}, \sqrt{p})$ is the Hilbert 2-class field over $\mathbb{Q}(\sqrt{2 p})$ (cf. [11]) and $\mathbb{Q}(\sqrt{2 p})$ is the maximal real subfield of the CM-field $\mathbb{Q}(\sqrt{-1}, \sqrt{2 p})$; so we let

$$
K=\mathbb{Q}(\sqrt{-1}, \sqrt{2 p}), \quad M=\mathbb{Q}(\sqrt{2}, \sqrt{p}) .
$$

The only prime ideal $(2, \sqrt{2 p})$ of $K^{+}=\mathbb{Q}(\sqrt{2 p})$ ramified in $K$ remains prime in $M$. Take any $\mathfrak{a} \in I_{M}$ satisfying $\mathfrak{a}^{2} \in I_{\mathbb{Q}}$. Clearly, $\mathfrak{a}$ is an ambiguous ideal for the quadratic extension $M / \mathbb{Q}(\sqrt{2 p})$. As $M$ is unramified over $\mathbb{Q}(\sqrt{2 p})$, $\mathfrak{a}$ has generators in $\mathbb{Q}(\sqrt{2 p}): \mathfrak{a} \in I_{\mathbb{Q}(\sqrt{2 p})}$. Therefore, in $I_{M}$,

$$
\mathfrak{a}=(\alpha) \text { or }(\sqrt{2})(\alpha) \quad \text { for some } \alpha \in \mathbb{Q}(\sqrt{2 p}) .
$$

Let $\varepsilon_{1}, \varepsilon_{2}$ and $\varepsilon_{3}$ denote respectively the fundamental units $>1$ of $\mathbb{Q}(\sqrt{2})$, $\mathbb{Q}(\sqrt{p})$ and $\mathbb{Q}(\sqrt{2 p})$ (so that $\varepsilon_{1}=1+\sqrt{2}$ ). Since

$$
N_{\mathbb{Q}(\sqrt{2}) / \mathbb{Q}}\left(\varepsilon_{1}\right)=N_{\mathbb{Q}(\sqrt{p}) / \mathbb{Q}}\left(\varepsilon_{2}\right)=N_{\mathbb{Q}(\sqrt{2 p}) / \mathbb{Q}}\left(\varepsilon_{3}\right)=-1,
$$

it now follows from Hilfssatz 6 of [8] that the three numbers $\sqrt{\varepsilon_{1} \varepsilon_{2} \varepsilon_{3}}, \varepsilon_{2}, \varepsilon_{3}$ form a system of fundamental units of $M$. Hence, by Hilfssatz 3 of [8], we easily have

$$
r\left(E_{M} / E_{M}^{*}\right)=4=[M: \mathbb{Q}] .
$$

The CM-field $K=\mathbb{Q}(\sqrt{-1}, \sqrt{2 p})$ thus satisfies the conditions (1-i)-(1-iii) of Theorem 1 . Hence, by Theorem $1, h_{K}^{-}$is odd, the Hilbert 2-class field over $K$ being $K M=\mathbb{Q}(\sqrt{-1}, \sqrt{2}, \sqrt{p})$.

The next lemma supplements Theorem 1 and will be useful to prove Theorem 2.

Lemma 2. Let $K$ be a $C M$-field and $M$ the Hilbert 2-class field over $K^{+}$. Assume that $2 \mid h_{K^{+}}, t_{K M}=1$, and hence $t_{K}=1$. Then the prime ideal of $K^{+}$ramified in $K$ divides 2 .

Proof. We take an algebraic integer $\alpha$ in $K^{+}$with $K=K^{+}(\sqrt{\alpha})$. Let $\mathfrak{p}$ be the unique prime ideal of $K^{+}$ramified in $K$, so that

$$
(\alpha)=\mathfrak{p}^{n} \mathfrak{a}^{2}
$$


for some integer $n \geq 0$ and for some integral ideal $\mathfrak{a}$ in $I_{K^{+}}$prime to $\mathfrak{p}$. It follows from $t_{K M}=1$ that $\mathfrak{p}$ remains prime in $M$. Hence, by class field theory, $A_{K^{+}}$is a cyclic group which is generated by the ideal class in $A_{K^{+}}$ containing the $\left(h_{K^{+}} /\left|A_{K^{+}}\right|\right)$-th power of $\mathfrak{p}$. Therefore $n$ is even. This conclusion shows that $\mathfrak{p}$ divides 2 , because $\mathfrak{p}$ must be unramified in $K$ if $\mathfrak{p}$ is prime to 2 .

As in the introduction, let $k$ be a totally real number field and let $\Gamma$ denote the set of all CM-fields $K$ with $K^{+}=k$. We fix $k$ from now on.

THEOREM 2. The following three statements are equivalent:

(2-i) $2 \nmid h_{k}$ and $r\left(E_{k} / E_{k}^{*}\right)=[k: \mathbb{Q}]$,

(2-ii) there exist infinitely many CM-fields in $\Gamma$ of odd relative class number,

(2-iii) there exist infinitely many $C M$-fields in $\Gamma$ of odd class number.

Pr o of. Clearly, (2-i) and (2-ii) imply (2-iii) while (2-iii) implies (2-ii). It therefore suffices to prove that $(2-\mathrm{i})$ is equivalent with (2-ii). Now, assuming (2-i), we let $\mathfrak{r}$ denote the product of distinct infinite primes of $k$. Let $c$ be the ideal class containing the principal ideal (3) in the ray class group of $k$ modulo (4)r. Let $\xi$ be any algebraic integer in $k$ such that $(\xi)$ is a prime ideal of $k$ in $c$. It follows that, for some $\varepsilon \in E_{k}, \varepsilon \xi$ is totally positive in $k$ and congruent to 3 modulo (4). Let $K=k(\sqrt{-\varepsilon \xi})$. Then $K$ is a CM-field contained in $\Gamma$ and $(\xi)$ is the unique prime ideal ramified in $K$. Hence Theorem 1 shows that $(2-i)$ implies $2 \nmid h_{K}^{-}$. Since there exist infinitely many prime ideals of $k$ in $c$, we can take infinitely many such CM-fields as $K$.

We next assume (2-ii), so that, by class field theory, there exists a CMfield in $\Gamma$ with odd relative class number in which a prime ideal of $k$ dividing an odd prime is ramified. We then have $2 \nmid h_{k}$ by Theorem 1 and Lemma 2 . Furthermore, by Theorem 1 , we also have $r\left(E_{k} / E_{k}^{*}\right)=[k: \mathbb{Q}]$. Thus $(2-\mathrm{i})$ follows from (2-ii).

Remark. As readily seen, (2-i) of Theorem 2 is equivalent to the condition that $h_{k}^{*}$ is odd.

For instance, suppose $k$ to be a real quadratic number field. Then, by Theorem 2, there exist infinitely many CM-fields in $\Gamma$ with odd relative class number if and only if $k=\mathbb{Q}(\sqrt{p})$ for some prime number $p \not \equiv-1(\bmod 4)$.

The following is an immediate consequence of Theorem 1.

Proposition 1. Let $L$ be the Hilbert 2-class field over $k$. Assume that $L$ is not cyclic over $k$ or $r\left(E_{L} / E_{L}^{*}\right) \leq[L: \mathbb{Q}]-2$. Then any CM-field in $\Gamma$ has even relative class number. 
2. Let $F$ be any number field. Then we denote by $F_{\infty}$ the basic $\mathbb{Z}_{2^{-}}$ extension over $F$; namely, we denote by $\mathbb{Q}_{\infty}$ the unique $\mathbb{Z}_{2}$-extension over $\mathbb{Q}$ in $\mathbb{C}$ and let $F_{\infty}=F \mathbb{Q}_{\infty}$ unless $F=\mathbb{Q}$. We write $\mu_{F}$ and $\lambda_{F}$ respectively for the Iwasawa $\mu$-invariant and the Iwasawa $\lambda$-invariant associated with the $\mathbb{Z}_{2}$-extension $F_{\infty} / F$. We further let $\tau(F)=1$ or 0 according as the ramification indices for $F_{\infty} / \mathbb{Q}_{\infty}$ of all primes of $F_{\infty}$ lying above 2 are even or not. Note that a prime of $F_{\infty}$ is ramified for $F_{\infty} / F$ if and only if the prime lies above 2. By the Hilbert 2-class field over $F$ in the narrow sense, we mean the maximal unramified abelian 2-extension over $F$ in which no prime ideal of $F$ is ramified. Now, for any CM-field $K$, let

$$
\mu_{K}^{-}=\mu_{K}-\mu_{K^{+}}, \quad \lambda_{K}^{-}=\lambda_{K}-\lambda_{K^{+}},
$$

and denote by $s_{K}$ the number of finite primes of $K_{\infty}^{+}=K^{+} \mathbb{Q}_{\infty}$ ramified in $K_{\infty}$ but not lying above 2 . It is easy to see $s_{K}<\infty$.

In this section, we shall prove:

Theorem 3. Let $k=k_{0} \subseteq \ldots \subseteq k_{n} \subseteq k_{n+1} \subseteq \ldots \subseteq k_{\infty}$ be the tower of intermediate fields of $k_{\infty} / k$ such that $\left[k_{n}: k\right]=2^{n}$ for each integer $n \geq 0$, let $m$ denote the maximal integer $\geq 0$ such that $k_{m} / k$ is unramified, and let $L^{*}$ denote the Hilbert 2-class field over $k$ in the narrow sense. Then there exist infinitely many CM-fields $K$ in $\Gamma$ with $\mu_{K}^{-}=\lambda_{K}^{-}=0$ if and only if the following conditions are satisfied:

(3-i) $h_{k_{m}}$ is odd,

(3-ii) $r\left(E_{k_{m}} / E_{k_{m}}^{*}\right)=\left[k_{m}: \mathbb{Q}\right]-\tau(k)$, i.e., $h_{k_{m}}^{*}=2^{\tau(k)} h_{k_{m}}$,

(3-iii) just one prime ideal of $L^{*}$ divides 2 .

For the proof, we prepare two lemmas.

Lemma 3. Let $F^{\prime} / F$ be a cyclic extension of number fields with 2-power degree such that just one prime ideal of $F$ is ramified in $F^{\prime}$. If $h_{F}^{*}$ is odd, then so is $h_{F^{\prime}}^{*}$.

Proof. Modifying the proof of II in [6], one can get a simple proof of this fact.

LEMma 4 (Corollary of Theorems 1 and 4 of [7]). Let $K$ be a CM-field such that $\mu_{K}^{-}=\lambda_{K}^{-}=0$. Then $s_{K}=1$ or 0 and, in the case $s_{K}=1$, just one prime of $K_{\infty}^{+}$lies above 2 .

Proof. Let $n$ be any integer $\geq 0$. Writing $K_{n}^{+}$for the intermediate field of $K_{\infty}^{+} / K^{+}$with degree $2^{n}$ over $K^{+}$, let $A_{n}^{*}$ denote the Sylow 2-subgroup of the ideal class group of $K_{n}^{+}$in the narrow sense. Let $\varrho$ be the number of primes of $K_{\infty}^{+}$lying above 2 . As $\mu_{K}^{-}=0$ implies $\mu_{K}=0$ (see, e.g., Proposition 13.24 of [12]), it then follows from Theorem 4 of [7] that

$$
r\left(A_{n}^{*}\right) \geq \varrho-1+\tau\left(K^{+}\right) \quad \text { if } n \text { is sufficiently large. }
$$


Furthermore, by Theorem 1 of [7],

$$
r\left(A_{n}^{*}\right)-\tau\left(K^{+}\right)-1+s_{K} \leq \lambda_{K}^{-}=0 \quad \text { if } n \text { is sufficiently large. }
$$

Hence we have $\varrho-1 \leq 1-s_{K}$, so that $s_{K}$ equals 1 or 0 and, in the case $s_{K}=1$, @ equals 1 .

Proof of Theorem 3. We denote by $\Omega$ the set of CM-fields $K$ in $\Gamma$ satisfying $\mu_{K}^{-}=\lambda_{K}^{-}=0$.

Let us first assume (3-i)-(3-iii). Since $L^{*} \supseteq k_{m}=k_{\infty} \cap L^{*}$, we see from (3-iii) that only a prime of $k_{\infty} L^{*}$ is ramified for $k_{\infty} L^{*} / L^{*}$ and it is totally ramified for $k_{\infty} L^{*} / L^{*}$. Furthermore, by (3-iii), $L^{*}$ is cyclic over $k$ so that $h_{L^{*}}^{*}$ is odd. Therefore, for any integer $n \geq m, h_{k_{n} L^{*}}^{*}$ is odd by Lemma 3 and hence $k_{n} L^{*}$ is the Hilbert 2-class field over $k_{n}$ in the narrow sense. We then also have

$$
\left[k_{n} L^{*}: k_{n}\right]=2^{\tau(k)}
$$

because (3-i) and (3-ii) imply $\left[L^{*}: k_{m}\right]=2^{\tau(k)}$ by class field theory. Let $\mathfrak{p}$ be any prime ideal of $k$ which does not divide 2 but remains prime in $k_{1}$. It follows that $\mathfrak{p}$ remains prime in $k_{n}$ for every integer $n \geq 0$. Let $\mathfrak{l}$ be the prime ideal of $k$ dividing 2 . As $\mathfrak{l}$ remains prime in $L^{*}$, we deduce from class field theory that $\mathfrak{p}^{a} \mathfrak{l}^{b}=(\omega)$ holds with an odd integer $a$, an integer $b$, and a totally positive element $\omega$ of $k$. Let

$$
J=k(\sqrt{-\omega}) .
$$

Obviously, $J$ is a CM-field in $\Gamma$ such that $s_{J}=1$ and hence $J_{\infty} \not \supset \sqrt{-1}$. Therefore, by Theorem 1 of [7] and by (2.1),

$$
\lambda_{J}^{-}=\tau(k)-\tau(k)=0 .
$$

Thus $J$ belongs to $\Omega$. It is now clear that $\Omega$ is an infinite set.

Assume next that $\Omega$ is infinite. Then $\Omega$ contains a CM-field $K$ in which a prime ideal of $k$ not dividing 2 is ramified, so that, by Lemma 4 ,

$$
s_{K}=1, \quad K_{\infty} \not \supset \sqrt{-1} .
$$

Let $j$ be any integer $\geq 0$, and let $L_{j}$ denote the Hilbert 2-class field over $k_{j}$. Note not only that $L_{j}$ is totally real but also that $L_{j} K$ is a CM-field. We find $s_{L_{j} K}=\left[L_{j} k_{\infty}: k_{\infty}\right]$ since the unique finite prime of $k_{\infty}$ ramified in $K_{\infty}$ and not lying above 2 is completely decomposed in $L_{j} k_{\infty}$. However, by Theorem 3 of [7], we have $\mu_{L_{j} K}^{-}=\lambda_{L_{j} K}^{-}=0$. Hence $L_{j} k_{\infty}=k_{\infty}$ follows from Lemma 4 . Consequently,

$$
L_{j}=k_{j} \quad \text { whenever } j \geq m .
$$

In particular, $h_{k_{m}}$ is odd. As just one prime of $k_{\infty}$ lies above 2 by Lemma 4 , there always exists a unique prime ideal of $L_{j}$ dividing 2 . Now, for each integer $n \geq 0$, let $L_{n}^{*}$ denote the Hilbert 2-class field over $k_{n}$ in the narrow 
sense. As the restriction map $\operatorname{Gal}\left(L_{j+1}^{*} / k_{j+1}\right) \rightarrow \operatorname{Gal}\left(L_{j}^{*} / k_{j}\right)$ is surjective in case $j \geq m$, we see from $\mu_{K}=0$ that

$$
r\left(\operatorname{Gal}\left(L_{j+1}^{*} / k_{j+1}\right)\right)=r\left(\operatorname{Gal}\left(L_{j}^{*} / k_{j}\right)\right) \quad \text { if } j \text { is sufficiently large. }
$$

Furthermore, by Theorem 1 of [7] and (2.2), we have

$$
r\left(\operatorname{Gal}\left(L_{j}^{*} / k_{j}\right)\right)=\tau(k) \quad \text { if } j \text { is sufficiently large. }
$$

It therefore follows from (2.3) and Lemma 3 that

$$
\left[L_{j}^{*}: k_{j}\right]=2^{\tau(k)} \quad \text { whenever } j \geq m .
$$

In particular, $h_{k_{m}}^{*}=2^{\tau(k)} h_{k_{m}}$. On the other hand, by Theorem 4 of [7], the unique prime ideal of $L_{j}$ dividing 2 remains prime in $L_{j}^{*}$ if $\tau(k)=1$ and $j$ is sufficiently large. Hence the prime ideal of $k$ dividing 2 remains prime in $L^{*}=L_{m}^{*}$. The conditions (3-i)-(3-iii) are thus satisfied and the proof is completed.

Corollary. There exist infinitely many $C M$-fields $K$ in $\Gamma$ with $\mu_{K}^{-}=$ $\lambda_{K}^{-}=0$ if and only if there exist infinitely many $C M$-fields $K$ in $\Gamma$ with $\mu_{K}=\lambda_{K}=0$.

Proof. In fact, if there exist infinitely many CM-fields $K$ in $\Gamma$ with $\mu_{K}^{-}=\lambda_{K}^{-}=0$, then we have $\mu_{k}=\lambda_{k}=0$ by Theorem 3 (cf. [6]).

Suppose $[k: \mathbb{Q}]=2$ in Theorem 3 . Then $m=0$ or 1 by genus theory. Furthermore, by Theorem 3, infinitely many CM-fields $K$ with $K^{+}=k$ satisfy $\mu_{K}^{-}=\lambda_{K}^{-}=0$ if and only if either $k=\mathbb{Q}(\sqrt{p})$ for some prime number $p \not \equiv 1(\bmod 8)$ or $k=\mathbb{Q}(\sqrt{2 p})$ for some prime number $p \equiv 5(\bmod 8)$ (cf. Example after Theorem 1). Here, in the first case, $m=0$ and

$$
\tau(k)= \begin{cases}0 & \text { if } p=2 \text { or } p \equiv 5(\bmod 8), \\ 1 & \text { if } p \equiv 3(\bmod 4) ;\end{cases}
$$

in the second case, $m=1$ and $\tau(k)=0$.

The following are almost immediate consequences of Theorem 1 of [7].

Proposition 2. Let $K$ be a $C M$-field. For each integer $n \geq 0$, let $K_{n}^{+}$ denote the intermediate field of $K_{\infty}^{+} / K^{+}$with degree $2^{n}$ over $K^{+}$and let $M_{n}^{*}$ denote the Hilbert 2-class field over $K_{n}^{+}$in the narrow sense. Then $\mu_{K}^{-}=\lambda_{K}^{-}=0$ if and only if

$$
r\left(\operatorname{Gal}\left(M_{n}^{*} / K_{n}^{+}\right)\right)+s_{K}+\delta=\tau\left(K^{+}\right)+1
$$

for every sufficiently large integer $n \geq 0$, where $\delta=1$ or 0 according as $K_{\infty}$ contains $\sqrt{-1}$ or not.

Proposition 3. Let $m$ and $k_{m}$ be the same as in Theorem 3. Assume that $r\left(A_{k_{m}}\right) \geq \tau(k)+2$ or $r\left(E_{k_{m}} / E_{k_{m}}^{*}\right) \leq\left[k_{m}: \mathbb{Q}\right]-\tau(k)-2$. Then either $\mu_{K}^{-}>0$ or $\lambda_{K}^{-}>0$ for any CM-field $K$ with $K^{+}=k$. 
3. We shall finally make a simple remark, omitting the details. Let $l$ be any odd prime and let $\mathbb{Z}_{l}$ denote the additive group of the $l$-adic integer ring. For each number field $F$, let $\mu_{l}(F)$ and $\lambda_{l}(F)$ denote respectively the Iwasawa $\mu$ - and $\lambda$-invariants associated with the basic $\mathbb{Z}_{l}$-extension over $F$. Let, for any CM-field $K$,

$$
\mu_{l}^{-}(K)=\mu_{l}(K)-\mu_{l}\left(K^{+}\right), \quad \lambda_{l}^{-}(K)=\lambda_{l}(K)-\lambda_{l}\left(K^{+}\right) .
$$

Then, as is well known, $K$ satisfies $\mu_{l}^{-}(K)=\lambda_{l}^{-}(K)=0$ if and only if $l$ neither divides $h_{K}^{-}$nor is divisible by any prime ideal of $K^{+}$decomposed in $K$ (see, e.g., Criterion 1.0 of [3]). We denote by $\Omega_{l}$ the set of CM-fields $K^{\prime}$ in $\Gamma$ for which $\mu_{l}^{-}\left(K^{\prime}\right)=\lambda_{l}^{-}\left(K^{\prime}\right)=0$.

Now, it seems quite likely that $\Omega_{l}$ is always an infinite set and hence there exist infinitely many CM-fields in $\Gamma$ with relative class number prime to $l$. This certainly holds in the case $l=3$; indeed, as Theorem 3 of [2] is refined by Theorem 1 of [10], so Theorem I.3 of [1] can be refined enough to show that a subset of $\Omega_{3}$ has a positive "density" in $\Gamma$ (cf. Proposition 2 and Theorem 3 of [10]). On the other hand, the main result of [9] states that $\Omega_{l}$ is an infinite set unless $l$ divides the non-zero integer $w \zeta_{k}(-1)$. Here $\zeta_{k}$ denotes the Dedekind zeta function of $k$ and

$$
w=2 \prod_{p} p^{n(p)},
$$

with $p$ ranging over the prime numbers ramified in $k(\sqrt{-1}, \sqrt{-3})$ and $n(p)$ denoting for each $p$ the maximal integer $\geq 0$ such that the $p^{n(p)}$-th roots of unity are contained in some quadratic extension over $k$. (For the special case where $k=\mathbb{Q}$, see also [5].)

\section{References}

[1] B. Datskovsky and D. J. Wright, Density of discriminants of cubic extensions, J. Reine Angew. Math. 386 (1988), 116-138.

[2] H. Daven port and H. Heilbronn, On the density of discriminants of cubic fields, II, Proc. Roy. Soc. London Ser. A 322 (1971), 405-420.

[3] E. Friedman, Iwasawa invariants, Math. Ann. 271 (1985), 13-30.

[4] H. Hasse, Bericht über neuere Untersuchungen und Probleme aus der Theorie der algebraischen Zahlkörper, Phisica-Verlag, Würzburg-Wien, 1970.

[5] K. Horie, A note on basic Iwasawa $\lambda$-invariants of imaginary quadratic fields, Invent. Math. 88 (1987), 31-38.

[6] K. Iw asawa, A note on class numbers of algebraic number fields, Abh. Math. Sem. Univ. Hamburg 20 (1956), 257-258.

[7] Y. Kida, Cyclotomic $\mathbb{Z}_{2}$-extensions of J-fields, J. Number Theory 14 (1982), 340352 .

[8] T. Kubota, Über den bizyklischen biquadratischen Zahlkörper, Nagoya Math. J. 10 (1956), 65-85. 
[9] H. Naito, Indivisibility of class numbers of totally imaginary quadratic extensions and their Iwasawa invariants, J. Math. Soc. Japan 43 (1991), 185-194.

[10] J. Nakagawa and K. Horie, Elliptic curves with no rational points, Proc. Amer. Math. Soc. 104 (1988), 20-24.

[11] L. Rédei and H. Reichardt, Die Anzahl der durch 4 teilbaren Invarianten der Klassengruppe eines beliebigen quadratischen Zahlkörpers, J. Reine Angew. Math. 170 (1933), 69-74.

[12] L. C. Washington, Introduction to Cyclotomic Fields, Springer, New York, 1982.

DEPARTMENT OF MATHEMATICS

TOKAI UNIVERSITY

HIRATSUKA 259-12

JAPAN

Received on 2.6.1993

and in revised form on 28.2.1994 